\title{
The COVID-19 Lockdown Revealed Implications for the Advancement of Behavioral Nudging Policies
}

\author{
Yudan Zhao ${ }^{1, a}$, Xiaoyue Zhang ${ }^{2, b}$
}

\author{
${ }^{1}$ University of Aberdeen \\ ${ }^{2}$ University of Aberdeen \\ ${ }^{a}$ Yudan Zhao.Email:t02ya20@abdn.ac.uk \\ ${ }^{b}$ Xiaoyue Zhang. Email: t08xz20@abdn.ac.uk
}

\begin{abstract}
The COVID-19 outbreak at the end of 2019 brought immeasurable damage to people worldwide, especially in China the first country to be attacked. The response has been a tough time for governments, health care workers, and the infected and uninfected who must be isolated. But with such a vast, live threatening pandemic threat, it is even more important to focus on the social and psychological factors that push people to behave, analyze and target research in a timely context so that the results can be better carried forward everyday life or policymaking. Because in this case, we can see more clearly than in ordinary life the drivers that are important to individuals. This contributes to our understanding of behavioral nudging and provides new directions for thinking and developing theoretical and practical applications.
\end{abstract}

Keywords: behavioral nudging, COVID-19, social influence

\section{INTRODUCTION}

Governments have long used critical or incentivebased approaches to regulate nations and cities' behavior, and these approaches are helpful. But in today's fastpaced era, more robust systems are needed to change citizens' behavior effectively. This has generated not only a great deal of research but also a need to explore new perspectives. Hence the emergence of a new path of inquiry into behavioral nudging. Through Change subtle aspects in daily life, such as speed limit signs that can be found everywhere in routine life; pedestrian crossings; reduced taxes on fruits and vegetables; limited or timelimited purchases or even music played in shopping malls. All of these unconsciously guide our behavior. Behavioral nudging can take many forms: mimicry, priming effects; authority; commitment; reciprocity, etc., which carries a dramatic role in social life. Considering that it can bring about significant changes in the behavior of a large proportion of the population with very little expense and wastage, it is not surprising that more attention has been given to the development of relevant policies. However, the specific implementation of behavioral inferences and the factors targeted should vary depending on culture, era, and the results of various research designs. Three types of behavioral changes and the possible driving factors behind them that emerged during the pandemic are discussed below.

\section{AUTHORITY}

Authority is one of the social influences of behavioral nudging, which occurs in two main ways: dominance and credibility. A typical example is the "Night of Shuanghuanglian". At the beginning of the outbreak in China, 'Xinhua Viewpoint' published on Weibo in the evening of January 31, 2020, "Chinese medicine SHL can inhibit coronavirus", which was re-posted by 'People's Daily'. As a result of the release of this information by two traditional reporting agencies, people queued up in front of pharmacies before the government had a clear idea of this new virus(Zhang, Chen, Jiang, \& Zhao, 2020). This increased the infection rate and placed an unnecessary burden on the government and hospitals. However, at this point governments does not have a complete understanding of the origin of the virus, its causation, or the drugs that can control the situation. The term "suppression" mentioned in this report refers to the progress of research on COVID-19 virus, and it seems that some of the components of SHL could contribute to the study. However, people abandoned their rational judgment in the face of tremendous pressure and just followed blindly, producing this farce. However, it is 
worth mentioning that no other drug except SHL has caused a similar hoarding frenzy, which is thy this case is used to emphasize the role of authority. There are three parties involved in this story. First, there is the role of dominance. As mentioned earlier, the two organizations have a high-level reputation in China, so the information they publish can be quickly disseminated and trustworthy. The second is credibility. In this article's title, the statements were made by a famous respiratory pathologist, Academician Zhong Nanshan. Therefore, people acted frantically without understanding the word "suppression" due to the vague headline.

There were, undoubtedly, other push factors involved. For example, sudden situations that lead tense of loss of control over one's life, especially the relatively aged and physically less healthy groups(Peluso \& Pichierri, 2020); vague representations of headlines; conformity behavior(Zhang et al., 2020), etc. Nonetheless, what is undeniable is the crucial contribution of authority in the event. Therefore, although it has gained researchers' attention, our next research direction should be how to control this force. When both dominance and credibility are high, people are more likely to believe these statements to save cognitive costs. Studies have shown that people will consider lies told by reputable experts to be more credible than the truth revealed by erevealedts with no reputation. Some researchers have hlly begun to think in this direction. In the United States and the United Kingdom, the government has already started to establish a particular supervisory institute, staffed by specialized experts, to explore the impact and application of behavioral nudging. This has proven to be a great success(Sunstein, 2014). In other countries, however, we also need to be mindful of the vast cultural differences in replication. One-size-fits-all cannot solve everything. Will government authority be greater in Asia or countries with collectivist cultures than in those with individualist cultures? Therefore behavioral nudges have to be advanced slowly, from the bottom up(Moseley \& Stoker, 2013). Because the impact on the group is too robust and hasty implementation may have serious consequences.

\section{MIMIC}

The spontaneous donation behavior of people during COVID-19 suggests insights for pushing more pro-social or charitable action. First, numerous changes in the environment and conditions have brought in many elements that lead to mimicry. In China, due to the national lockdown, with more availability of time for themselves, they have seen a rapid increase in screen time, especially in social media(Bann et al., 2020). Also, without direct, face-to-face contact with the outside world, attention to information about the outbreak was likewise limited to social networking platforms. Thus, significant exposure to healthcare workers' hard work working in the first and second line. Presumably, healthcare workers' life-saving role at this particular time has generated widespread respect, trust, and praise. And predisposed liking emotions, affiliated goals can cause mimicry behaviors(Chartrand \& Lakin, 2013). Therefore, it can be hypothesized that many charitable acts emerge as an emotional imitation of frontline staff by the population when faced with these videos, pictures, and news reports. Through this emotional contagion, it is possible that even people far away from the infected area can empathize with such mimicry and thus facilitate the emergence of helping behaviors.

Until April 23, 2020, China's national charitable organizations and the Red Cross received about 40000 billion yuan in donations from all works of life; about 1000 billion pieces of donated materials. Although the reasons behind what drove the emergence of this behavior need to be supported by specific statistics, it is time to re-introduce attention to promoting emotional mimicry approaches. It is worth noting that behavioral nudging is best carried out when there is no awareness (Halpern, 2015). When people recognize that manipulative behavior is behind these changes, they engage in conscious control. We, therefore, need to examine how we can expand the role of charity advertising naturally. On Taobao ---- a Chinese shopping app - searching for the most expensive thing yields a donation screen. This is a recommendation from a user I saw while browsing on social media platforms. Whether this is a government effort or not is not clear to me. But after seeing this message and doing a search, my friend and I both made partial donations. Of course, it will take a lot of statistical proof and, as mentioned above, slow progress to apply it in practice.

\section{SIMPLE}

So far, uncountable rumors have emerged across the globe. The power of these rumors is so strong, especially in the early stages of the epidemic. For example, the "SHL night" described at the beginning of the paper, "A sow speaks and claim that eating nine eggs will prevent the epidemic" and "Food shortage suggests stocking up on food". Like the analysis on the previous section, increasingly available time and limited space - with uninfected people being isolated at home and infected people having even less space at their disposal, with only a few square meters of ward space - keeps people informed only online, through national, media, etc. news releases and messages from friends and relatives. That dramatically raises the likelihood that persons will be vulnerable to rumors. Even now, many words grab attention, such as "the time of unsealing everywhere is determined" and "the time of opening of colleges is determined". Given the extended lockdown, these rumors, which seem to have reliable sources and are concise and clear, are widely spread and cause serious consequences, adding to the government's burden in all 
aspects. The epidemic, in reality, has been partially controlled, but the misinformation outbreak is still fermenting. It is critical to think about the reasons behind these confidence behaviors. Because it can tell us the reason why these rumors are being highly publicized, minimizing the damage. It can also be applied to the delivery of correct information and boosting trust.

What are the reasons that rumors are widely spread and trusted? In every story, misinformation has one common feature, brevity and clarity. Analyzing from the "SHL night", the headline brings an explicit instruction to the masses that taking the drug Shuanghuanglian can get rid of the threat to life. It seems that in an uncertain situation, following the simple instructions forwarded by the government or someone close to them is the wisest thing to do. To help themselves and those close to them regain a sense of control over their own lives and return to stability within their inner world, people will not only choose to believe rumors but also accelerate its spread. "Make it easy" is a fundamental principle that governments or organizations should follow when delivering messages. In the age of information explosion, helping residents reduce cognitive stress and get as much accurate information as possible is something that the government needs to consider. A U.S. study demonstrated a statistically significant increase in high school student enrollment when a more streamlined approach was used for assistance (Eric P. et al., 2019). Therefore, at this critical moment, it is essential to increase the regulation of information sent on the Internet and the legal requirements; it is more important to "make it easy" the way authoritative information is released.

\section{DISCUSSION}

In analyzing the circumstances that occurred during the COVID-19 outbreak, it can be observed that the three factors mentioned in this paper are intertwined and interact with each other. Authoritative institutions or experts with high credibility can generate public liking and respect. Establishing practical policies, constantly exploring research in one's field, and promoting the quality of life with happiness of global citizens based on the results of the investigation can create affiliation purposes among the public. As mentioned in the imitation section, these factors can significantly promote the mimetic behavior of the masses, driving them to adopt and exercise healthier lifestyles; carry out the responsibilities and obligations set by the government, etc. The literature mentioned in the simplicity panel also proves the importance of simple guidance in guiding behavior by the government. However, whether these factors explored in this paper play significant prominent role needs further experimental proof. However, whether these factors discussed in this paper play such a significant role requires more empirical evidence proof. How to apply or manage these interactions needs to be continuously discovered. Consider the complexity and diversity of human behavior.

Nonetheless, nudges will continue to be an effective way in inducing specific behavioral change in particular specific settings. These approaches can complement traditional models, such as heavier punishment and deterrence, and reward positive behavior. But they cannot replace laws.

Subsequent studies should also focus on adaptive behaviors that people exhibit during this unique period. Examples include utilizing limited resources for physical activity at home (Results from 5 UK cohort studies show); cooking meals with more healthy ingredients; and increased hand washing as well as disinfection after going to public places(Weijers \& de Koning, 2020). Via the behavioral nudges mentioned in this paper, it can be hypothesized that (a) professional advice from the government and reputable physicians has contributed to the increase in disinfection behaviors; and (b) the growing number of daily sharing or teachings posted on social media has provided a way for people to find pleasure in their lives. The desire to feel this way promotes the emergence of mimicry; (c) it is more accessible and more common sense to use medical alcohol for sterilization in the early stages of a viral epidemic as opposed to the hopeless act of lockdown. Analysis of this type of behavior has great applied value. Although many positive behaviors were promoted during this particular period, when this period has passed, more attention is needed to identify the factors that could sustain these actions for the masses. Hume, John, Sanders \& Stockdale, published a paper in 2020 reporting on an online experiment they conducted. The results showed that when victims performed a reflective task, it changed their intention to follow the rules, but lasted only two weeks. Consequently, focusing only on the cause itself is not sufficient, how to maximize the effect and impacting the effect to the greatest extent is the direction in which we need to put our eyes on more.

\section{CONCLUSION}

While the sudden outbreak of COVID-19 has caused a lot of harm, both socially and personally, we need to capture this opportunity to notice the aspects that have been neglected before or need to be improved in line with the times and keep promoting social progress and creating a harmonious social environment. In particular, factors such as authority, imitation, and simplicity can significantly contribute to crisis time crisis. However, it is still necessary to remind again that this cannot be directly analogized to different cultural environments. Once again, the reminder is not onerous, the development of behavioral nudge policies needs to be approached slowly and cannot be rushed. In addition, results in one country or culture cannot be directly replicated in other countries; different cultures, ethnicities, and socio-economic status of the nationals need to be taken into account and replicated while 
controlling for risk factors. Therefore, the application of behavioral nudges needs further refinement and polishing, constant refinement of research, and cautious experimentation.

\section{REFERENCES}

[1] Bann, D., Villadsen, A., Maddock, J., Hughes, A., Ploubidis, G. B., Silverwood, R. J., \& Patalay, P. (2020). doi:10.1101/2020.07.29.20164244

[2] Changes in the behavioural determinants of health during the coronavirus (COVID-19) pandemic: gender, socioeconomic and ethnic inequalities in 5 British cohort studies (Updated September 17, 2020). (2020). In Medical Letter on the CDC \& FDA (p. 206-). NewsRX LLC.

[3] Chartrand, T. L., \& Lakin, J. L. (2013). The antecedents and consequences of human behavioral mimicry. Annu Rev Psychol, 64, 285-308. doi:10.1146/annual-psych-113011-143754

[4] Eric P. Bettinger \& Bridget Terry Long \& Philip Oreopoulos \& Lisa Sanbonmatsu, 2009. "The Role of Simplification and Information in College Decisions: Results from the H\&R Block FAFSA Experiment," NBER Working Papers 15361, National Bureau of Economic Research, Inc.

[5] Halpern, D. (2015). Inside the Nudge Unit: How small changes can make a big difference: Ebury Publishing.
[6] Hume, S., John, P., Sanders, M., \& Stockdale, E. (2020). Nudge in the Time of Coronavirus: The Persistence of Behavioural Messages during Crisis. SSRN Electronic Journal. doi: 10.2139/ssrn.3644165

[7] Moseley, A., \& Stoker, G. (2013). Nudging citizens? Prospects and pitfalls were confronting a new heuristic. Resources, Conservation and Recycling, 79, 4-10. doi:10.1016/j.resconrec.2013.04.008

[8] Peluso, A. M., \& Pichierri, M. (2020). Effects of socio-demographics, sense of control, and uncertainty avoidability on post-COVID-19 vacation intention. Current Issues in Tourism, 1-13. doi:10.1080/13683500.2020.1849050

[9] Sunstein, C. R. (2014). Nudging: A Very Short Guide. Journal of Consumer Policy, 37(4), 583-588. doi:10.1007/s10603-014-9273-1

[10] Weijers, R., \& de Koning, B. (2020). Nudging to increase hand hygiene during the COVID-19 pandemic: A field experiment. Canadian Journal Of Behavioural Science / Revue Canadienne Des Sciences Du Comportement. doi: $10.1037 / \mathrm{cbs} 0000245$

[11] Zhang, L., Chen, K., Jiang, H., \& Zhao, J. (2020). How the Health Rumor Misleads People's Perception in a Public Health Emergency: Lessons from a Purchase Craze during the COVID-19 Outbreak in China. Int J Environ Res Public Health, 17(19). doi:10.3390/ijerph17197213 